\title{
Hipotermia perioperatória: conhecimentos e intervenções da equipe de enfermagem
}

\begin{abstract}
RESUMO | Objetivo: Avaliar o conhecimento e intervenções da equipe de enfermagem sobre hipotermia perioperatória no paciente cirúrgico. Método: Estudo descritivo-exploratório com corte transversal de abordagem quantitativa. Foi realizada a coleta dos dados com 77 profissionais entre enfermeiros e técnicos de enfermagem atuantes no bloco operatório e da sala de recuperação pós-anestésica de um hospital de referência em cirurgias de alta complexidade. Resultados :A pesquisa evidencia que os participantes obtêm conhecimento básico sobre o assunto, principalmente sobre o conceito, os sinais, os sintomas e como intervir quando o paciente apresenta o problema. As complicações e o seu manejo apresentam-se como uma dificuldade. Conclusão: O conhecimento sobre hipotermia perioperatória e as intervenções da equipe enfermagem apresenta lacunas. Esperase contribuir no planejamento de ações educativas que norteiem as estratégias de assistência de qualidade ao paciente cirúrgico. Palavras-chaves: Hipotermia; Enfermagem Perioperatória; Centro Cirúrgico; Complicações Intraoperatórias.
\end{abstract}

\begin{abstract}
Objective: To evaluate the knowledge and interventions of the nursing team about perioperative hypothermia in surgical patients. Method: Descriptive-exploratory study with a cross-sectional approach using a quantitative approach. Data were collected from 77 professionals, including nurses and nursing technicians working in the operating room and in the postanesthetic recovery room of a reference hospital in overly complex surgeries. Results: The research shows that the participants obtain basic knowledge about the subject, mainly about the concept, the signs, the symptoms and how to intervene when the patient presents the problem. Complications and their management present themselves as a difficulty. Conclusion: The knowledge about perioperative hypothermia and the interventions of the nursing team has gaps. It is expected to contribute to the planning of educational actions that guide quality care strategies for surgical patients.
\end{abstract}

Keywords: Hypothermia; Perioperative Nursing; Surgical Center; Intraoperative Complications.

RESUMEN | Objetivo: evaluar el conocimiento y las intervenciones del equipo de enfermería sobre la hipotermia perioperatoria en pacientes quirúrgicos. Método: estudio descriptivo-exploratorio con un enfoque transversal utilizando un enfoque cuantitativo. La recopilación de datos se realizó con 77 profesionales, incluidas enfermeras y técnicos de enfermería que trabajan en la sala de operaciones y en la sala de recuperación postanestésica de un hospital de referencia en cirugías altamente complejas. Resultados: La investigación muestra que los participantes obtienen conocimientos básicos sobre el tema, principalmente sobre el concepto, los signos, los síntomas y cómo intervenir cuando el paciente presenta el problema. Las complicaciones y su manejo se presentan como una dificultad. Conclusión: El conocimiento sobre la hipotermia perioperatoria y las intervenciones del equipo de enfermería tiene lagunas. Se espera que contribuya a la planificación de acciones educativas que guíen las estrategias de atención de calidad para pacientes quirúrgicos.

Descriptores: Hipotermia; Enfermería Perioperatoria; Centro Quirúrgico; Complicaciones Intraoperatorias.

Emanuela Batista Ferreira e Pereira

Doutora em Cirurgia. Universidade de Pernambuco - UPE, Recife, PE.

\section{Fernanda da Mata Vasconcelos Silva}

Doutoranda em Enfermagem. Universidade de Pernambuco - UPE, Recife, PE.

\section{Fabiana Nascimento Mendes}

Enfermeira. Governo do Estado de Pernambuco, Recife, PE.

\section{José Almir Alves da Silva}

Graduando de Enfermagem. Universidade Maurício de Nassau Caruaru, PE.

\section{Maria Sonja Oliveira de Oliveira}

Enfermeira. Faculdade de Ciências Humanas de Olinda - FACHO Olinda, PE.

\section{Roberto Bezerra da Silva}

Doutor em Terapia Intensiva. Hospital do Câncer de Pernambuco -HCP, Recife, PE.

Recebido em: 26/04/2020

Aprovado em: 27/04/2020

INTRODUÇÃO

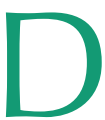
urante o procedimento cirúrgico, o paciente está sujeito a alguns fatores de risco, dentre eles, a hipotermia, que se caracteriza por um estado clínico em que a temperatura corporal se encontra abaixo de $36^{\circ} \mathrm{C}^{(1)}$. Tendo o hipotálamo como centro termorregulador, a temperatura corporal é um dos parâmetros fisiológicos rigorosamente controlados do nosso organismo, e um dos responsáveis pelo seu perfeito funcionamento que aceita variações na faixa de $36,4^{\circ} \mathrm{C}$ a $37,6^{\circ} \mathrm{C}^{(2,3)}$.
O corpo humano pode perder calor de quatro formas: radiação, convecção, condução e evaporação. A radiação promove a perda de calor por ondas eletromagnéticas, quando o corpo apresenta temperatura maior que o ambiente. A perda por convecção ocorre quando a diferença de densidade da água ou do ar se transfere para o corpo, removendo o calor superficial da pele. Na perda por condução, o calor é perdido por contato direto, havendo uma transferência do calor corporal para um objeto com temperatura mais baixa. Enquanto na evaporação, ocorre a difusão dos líquidos presentes na pele para vapor no ambiente, o que ocorre na respiração e na sudorese $\mathrm{e}^{(3)}$.

Através dessas formas de perda de calor, pode ocorrer a hipotermia, um quadro clínico em que o organismo não 
é capaz de gerar o calor necessário para garantir a adequada manutenção das funções fisiológicas. A hipotermia pode ser considerada leve $\left(32^{\circ}\right.$ a $\left.35^{\circ} \mathrm{C}\right)$, moderada $\left(28^{\circ}\right.$ a $\left.32^{\circ} \mathrm{C}\right)$ e grave - menor que $28^{\circ} \mathrm{C}^{(4)}$.

Essa condição pode afetar o metabolismo, acarretando diversas complicações, tais como: risco de infecção do sítio cirúrgico, alterações da função renal, distúrbios de coagulação, rebaixamento do nível de consciência e consequentemente, aumento do tempo de internação ${ }^{(5)}$.

O ato cirúrgico geralmente é responsável por promover a hipotermia no paciente devido a diversos fatores, como a indução anestésica que tem ação vasodilatadora e por reduzir o metabolismo e interferir com o centro termorregulador central ${ }^{(6)}$.

Outros fatores como o tempo e o tipo de cirurgia, a exposição das cavidades corporais às baixas temperaturas mantidas nas salas operatórias (entre 19 e $24{ }^{\circ} \mathrm{C}$ ), as alterações corporais causadas pela infusão de líquidos e hemoderivados frios, agentes anestésicos, assim como também preparações para a pele frias ${ }^{(1)}$. A própria condição do paciente também pode ser um fator de risco que o predispõe a hipotermia: extremos de idade e de peso corporal, distúrbios neurológicos e doenças metabólicas ${ }^{(7)}$.

Estudos mostram que, quando não induzida, a hipotermia ocorre em mais de $70 \%$ dos pacientes que são submetidos a procedimentos anestésico-cirúrgicos, geralmente nos períodos intra e pós-operatório ${ }^{(8)}$.

Quanto aos sinais e complicações, observa-se que o paciente hipotérmico pode apresentar tremores, extremidades frias, cianose, piloereção, diminuição do metabolismo, função renal alterada, alteração do nível de consciência, depressão respiratória e até mesmo coagulopatias ${ }^{(9)}$.

Para minimizar a ocorrência deste agravo é importante que a equipe cirúrgica, e em especial a de enfermagem, atue na promoção de medidas para manutenção da normotermia ao paciente cirúrgico e, por conseguinte, a prevenção das complicações $^{(10)}$.
Dessa forma, é importante a monitorização da temperatura corporal dos pacientes no intra e pós-operatório para facilitar o controle térmico e evitar as possíveis complicações causadas pela hipotermia. Nesse sentido, a enfermagem destaca-se como um dos principais agentes na redução de possíveis complicações causadas pela hipotermia.

Ao enfatizar a importância da atuação da equipe de enfermagem, seu conhecimento e intervenções na monitorização e manutenção da temperatura corporal dentro dos níveis de segurança do paciente durante o período perioperatório justifica-se a relevância deste estudo que visa contribuir para um procedimento cirúrgico seguro e com resultados satisfatórios.

Diante do exposto, pretende-se responder o seguinte questionamento: $\mathrm{O}$ que os enfermeiros e técnicos de enfermagem sabem sobre hipotermia perioperatória e como atuam para prevenir e combatê-la? Assim, o objetivo do presente estudo foi avaliar o conhecimento da equipe de enfermagem do bloco operatório (BO) e da sala de recuperação pós-anestésica (SRPA) sobre hipotermia no paciente perioperatório.

\section{METODOLOGIA}

Trata se de um estudo descritivo-exploratório com corte transversal de abordagem quantitativa.

A coleta dos dados ocorreu no período de julho a setembro de 2017 no centro cirúrgico de um hospital de referência do Estado de Pernambuco. Com estratégia de busca pelos sujeitos da pesquisa foi realizada a abordagem pessoalmente do profissional e apresentado o objetivo da pesquisa e a necessidade da assinatura do Termo de Consentimento Livre e Esclarecido.

Após o recebimento das duas vias, leitura e a assinatura do Termo de Consentimento Livre e Esclarecido, os participantes responderam a um formulário semiestruturado autoaplicável, adaptado pelas pesquisadoras, do instrumento uti- lizado no estudo de Mendonza ${ }^{(11)}$, contendo seis questões relacionadas aos aspectos sociodemográficas e nove ao conhecimento sobre a ocorrência de hipotermia no paciente perioperatório.

Ressalta-se que o desenvolvimento da pesquisa atendeu às normas nacionais e internacionais de ética em pesquisa envolvendo os seres humanos e a coleta dos dados foi realizada após a aprovação pelo Comitê de Ética e Pesquisa (CEP) da Universidade de Pernambuco sob o número do parecer: 69052317.8.0000.5198.

Como critérios de inclusão foram estabelecidos que os participantes fossem enfermeiros ou técnicos de enfermagem atuantes há pelo menos 3 meses no centro cirúrgico ou na sala de recuperação pós-anestésica que se disponibilizaram a participar da pesquisa. Foram excluídos os colaboradores que tivessem realizado algum treinamento sobre prevenção de hipotermia perioperatória nos últimos 3 meses, e os que estavam de licença médica e férias no período da coleta de dados.

A amostra dos participantes foi não probabilística por adesão sendo composta por 77 funcionários entre enfermeiros e técnicos de enfermagem atuantes no bloco operatório e na sala de recuperação pós-anestésica.

Para análise dos dados foi construído um banco em planilha eletrônica no programa Microsoft Excel a qual foi exportada para o Software Estatístico SPSS version $21^{\circledR}$, onde foi realizada a associação entre as variáveis quantitativas dos dados coletados. Foram calculadas as frequências percentuais para avaliar o perfil sociodemográfico da população participante do estudo, a descrição do conhecimento da equipe da equipe de enfermagem identificando as práticas e estratégias utilizadas para o manejo da hipotermia perioperatória.

Ainda, foi aplicado o teste do Qui-quadrado de comparação de proporção para comparar os percentuais encontrados nos níveis dos fatores avaliados. Esse teste considerou satisfatórias as conclusões com o nível de significância de 5\%. 
Os resultados foram agrupados em tabelas, posteriormente, discutidos à luz da literatura científica.

\section{RESULTADOS}

Na Tabela 1 temos a distribuição do perfil pessoal dos profissionais avaliados. Verifica-se que a maioria composta por técnicos de enfermagem $(72,7 \%)$, é do sexo feminino $(86,2 \%)$, possui idade de 31 a 45 anos (47,0\%), atua profissionalmente a mais de 15 anos $(41,6 \%)$, sendo que 53,5\% no centro cirúrgico e não tiveram nenhuma capacitação sobre hipotermia perioperatória $(92,2 \%)$. O teste de comparação de proporção foi significativo em todos os fatores avaliados ( $\mathrm{p}$-valor menor do que 0,05$)$, indicando que o perfil descrito é relevantemente o mais presente nos profissionais avaliados. Em média, o grupo possui idade de 41,9 anos com desvio padrão de 11,1 anos. Para o tempo de atuação, a média foi de 14,6 anos com desvio de 11,0 anos, e para o tempo de atuação no centro cirúrgico, a média foi de 8,4 anos com desvio padrão de 9,1 anos.

\section{Tabela 1. Distribuição do perfil pessoal dos profissionais avaliados. Olinda, PE, Brasil, 2017}

\section{Fator avaliado}

Q1 - Categoria profissional

Enfermeiro

Técnico de enfermagem

Q2 - Sexo

Masculino

Feminino

Q3 - Idade
N

21

56
$\%$

27,3

72,7

\section{\%}

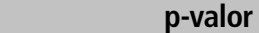

22 a 30 anos

9

13,8

$<0,001$

31 a 45 anos

56

86,2

46 a 59 anos

Igual ou maior a 60 anos

Mínimo - Máximo

Média \pm Desvio padrão

Q4 - Tempo de atuação profissional

\begin{tabular}{|c|c|c|c|}
\hline Até 5 anos & 22 & 28,6 & 0,002 \\
\hline 11 a 15 anos & 13 & 16,9 & \\
\hline Mais de 15 anos & 32 & 41,6 & \\
\hline Mínimo - Máximo & $0,25-40,0$ & - & \\
\hline Até 5 anos & 38 & 53,5 & $<0,001$ \\
\hline 6 a 10 anos & 12 & 16,9 & \\
\hline 11 a 15 anos & 9 & 12,7 & \\
\hline 60 ou mais anos & 12 & 16,9 & \\
\hline \multicolumn{4}{|c|}{ Q6 - Já teve alguma capacitação sobre hipotermia perioperatória? } \\
\hline $\operatorname{Sim}$ & 6 & 7,8 & $<0,001$ \\
\hline Não & 71 & 92,2 & \\
\hline
\end{tabular}

Nota: ${ }^{1} p$-valor do teste de comparação de proporção. 
Na Tabela 2 temos a distribuição dos fatores relacionados ao conhecimento dos profissionais acerca da Hipotermia. A maioria dos profissionais avaliados afir- maram que a hipotermia é a baixa temperatura corporal $(98,7 \%)$, considera que o Hipotálamo é o centro termorregulador da temperatura corporal $(79,6 \%)$, acredita que a evaporação é a principal forma de perda de calor $(37,0 \%)$ e considera que em todos os momentos, pré, durante e pós operatório, é importante realizar o controle de

Tabela 2. Distribuição dos fatores relacionados ao conhecimento dos profissionais acerca da hipotermia. Olinda, PE, Brasil, 2017

\begin{tabular}{lcc} 
Fator avaliado & N & p-valor ${ }^{1}$ \\
Q7 - O que é Hipotermia & 76 & 98,7 \\
\hline Baixa temperatura corporal & 1,3 & 1,3
\end{tabular}

Q8 - Qual o centro termorregulador da temperatura corporal

\begin{tabular}{|c|c|c|c|}
\hline Hipotálamo & 66 & 79,6 & $<0,001$ \\
\hline Hipófise & 8 & 9,6 & \\
\hline Bulbo & 8 & 9,6 & \\
\hline \multicolumn{4}{|l|}{ Q9 - Formas de perda de calor } \\
\hline Evaporação & 37 & 37 & $<0,001$ \\
\hline Condução & 11 & 11 & \\
\hline Irradiação & 9 & 9 & \\
\hline Outros & 18 & 18 & \\
\hline \multicolumn{4}{|c|}{$\begin{array}{l}\text { Q10 - Em quais momentos é importante } 0 \text { - realizar o controle da } \\
\text { temperatura corporal do paciente no Centro Cirúrgico? }\end{array}$} \\
\hline Após o procedimento cirúrgico & 5 & 7,1 & \\
\hline Em todos os momentos, pré, durante e pós-cirúrgico & 32 & 45,7 & \\
\hline No momento pré e pós-cirúrgico & 5 & 7,1 & \\
\hline Durante e pós-cirúrgico & 7 & 10,0 & \\
\hline
\end{tabular}

Nota: ${ }^{1} \mathrm{p}$-valor do teste de comparação de proporção.

Tabela 3. Distribuição dos sinais que indicam a presença de hipotermia operatória. Olinda, PE, Brasil, 2017

\begin{tabular}{lcc|}
\hline Fator avaliado & N & $\%$ \\
\hline Tremores & 36 & 20,7 \\
\hline Cianose & 29 & 16,8 \\
\hline Pele fria & 19 & 10,9 \\
\hline Extremidades frias & 12 & 6,8 \\
\hline Calafrios & 11 & 6,3 \\
\hline Hipotensão & 10 & 5,7 \\
\hline Sudorese & 7 & 4,0 \\
\hline Taquicardia & 4 & 2,3 \\
\hline Bradicardia & 3 & 1,7 \\
\hline Baixa saturação & 2 & 1,2
\end{tabular}

temperatura corporal do paciente $(45,7 \%)$. O teste de comparação de proporção foi significativo em todos os fatores avaliados, indicando que a descrição realizada sobre o conhecimento dos profissionais é, relevantemente, o mais presente.

$\mathrm{Na}$ Tabela 3 temos a distribuição das respostas dos profissionais avaliados acerca dos sinais que indicam a presença de hipotermia operatória. Verifica-se que os sinais mais citados foram: tremores $(20,7 \%)$, cianose $(16,8 \%)$, pele fria $(10,9 \%)$, extremidade frias $(6,8 \%)$, calafrios $(6,3 \%)$ e hipotensão $(5,7 \%)$.

Na Tabela 4 temos a distribuição dos fatores relacionados aos pacientes, ao ambien- 


\begin{tabular}{|lcc|}
\hline Baixa temperatura & 2 & 1,2 \\
\hline Bradipneia & 2 & 1,2 \\
\hline Sangramento & 2 & 1,2 \\
\hline Temperatura abaixo de $34^{\circ}$ & 2 & 1,2 \\
\hline Hemorragia & 2 & 1,2 \\
\hline Palidez & 2 & 1,2 \\
\hline Pulso fraco & 2 & 1,2 \\
\hline Queda de pressão & 2 & 1,2 \\
\hline Suor & 2 & 1,2 \\
\hline Sonolência & 2 & 1,2 \\
\hline Outros* & 20 & 11,6 \\
\hline
\end{tabular}

Nota: *Outros sinais com frequência unitária.

Tabela 4. Distribuição dos fatores que podem contribuir para a ocorrência da hipotermia perioperatória. Olinda, PE, Brasil, 2017

\begin{tabular}{lcc|} 
Fator avaliado & N & $\%$ \\
\hline Tipo de anestesia & 46 & 24,5 \\
\hline Tempo da cirurgia & 45 & 23,9 \\
\hline Idade & 45 & 23,9 \\
\hline Tipo de cirurgia & 40 & 21,3 \\
\hline Outros & 12 & 6,4
\end{tabular}

Tabela 5. Distribuição dos métodos conhecidos pelos profissionais para aquecer o paciente. Olinda, PE, Brasil, 2017

\begin{tabular}{lcc} 
Fator avaliado & N & $\%$ \\
\hline Aquecedor & 59 & 35,5 \\
\hline Colchão/manta térmico & 47 & 28,3 \\
\hline Soro aquecido & 28 & 16,9 \\
\hline Cobertores & 25 & 15,1 \\
\hline Temperatura do ambiente & 4 & 2,4 \\
\hline Algodão ortopédico & 1 & 0,6 \\
\hline Ataduras & 1 & 0,6 \\
\hline Botas & 1 & 0,6
\end{tabular}

Tabela 6. Distribuiç̧ão da prática realizada durante a ocorrência de hipotermia. Olinda, PE, Brasil, 2017

\begin{tabular}{lcc} 
Fator avaliado & N & $\%$ \\
\hline Aquece o paciente & 65 & 58,6 \\
\hline Aplica soro morno & 35 & 31,5 \\
\hline Desliga o ar condicionado & 6 & 5,4 \\
\hline Aplica medicamentos & 5 & 4,5
\end{tabular}

Tabela 7. Distribuição das possíveis complicações decorrentes da hipotermia perioperatória. Olinda, PE, Brasil, 2017

Fator avaliado N te e ao procedimento cirúrgico que podem contribuir para a ocorrência da hipotermia perioperatória. Verifica-se que os fatores mais citados pelos profissionais foram: o tipo de anestesia $(24,5 \%)$, o tempo de cirurgia $(23,9 \%)$ e a idade do paciente $(23,9 \%)$.

Na Tabela 5 temos a distribuição dos métodos conhecidos para aquecer o paciente. Verifica-se que as práticas mais conhecidas são: uso de aquecedor (35,5\%), colchão/manta térmica $(28,3 \%)$, soro aquecido (16,9\%) e cobertores (15,1\%). Ainda, observa-se que os métodos menos citados foram: botas, ataduras e algodão ortopédico (ambos com 0,6\%).

Na Tabela 6 temos a distribuição das medidas adotadas quando o paciente apresenta hipotermia no centro cirúrgico. Verifica-se que aquecer o paciente e aplicar soro aquecido são os procedimentos adotados pela maioria dos profissionais avaliados.

Na Tabela 7 temos a possíveis complicações decorrentes da hipotermia perioperatória citadas pelos profissionais avaliados. Verifica-se que as consequências mais relatadas são: óbito $(9,4 \%)$, parada cardíaca $(8,2 \%)$, hipotensão $(6,3 \%)$, choque $(6,3 \%)$ e tremedeiras $(5,0 \%)$.

\section{DISCUSSÃO}

Os resultados da presente pesquisa mostram que o sexo feminino ainda é uma característica predominante na enfermagem. A maioria dos entrevistados não teve nenhuma capacitação sobre o assunto, porém demonstra conhecimento sobre o conceito de hipotermia e sobre o hipotálamo ser o centro responsável pela termorregulação.

A média de idade dos entrevistados e seu tempo de atuação em centro cirúrgico mostram que a experiência prática provavelmente contribui para o aumento do conhecimento desses profissionais, mas ainda há a necessidade de enfatizar a importância da educação permanente com a equipe de saúde.

Quanto a outros aspectos abordados sobre a hipotermia, sobre as formas de perda de calor pelo corpo, muitos profissionais desconhecem as outras três formas que são a condução, convecção e irradiação, sendo a última a 


\begin{tabular}{|lll|}
\hline Óbito & 15 & 9,4 \\
\hline Parada cardiorrespiratória & 13 & 8,2 \\
\hline Hipotensão & 10 & 6,3 \\
\hline Choque & 10 & 6,3 \\
\hline Tremedeiras & 8 & 5,0 \\
\hline Bradicardia & 7 & 4,4 \\
\hline Cianose & 5 & 3,1 \\
\hline Arritmia & 4 & 2,5 \\
\hline Aumento das chances de infecção & 4 & 2,5 \\
\hline Coagulopatias & 4 & 2,5 \\
\hline Calafrios & 3 & 1,9 \\
\hline Taquicardia & 3 & 1,9 \\
\hline Convulsões & 3 & 1,9 \\
\hline Hemorragia & 3 & 1,9 \\
\hline Isquemia & 3 & 1,9 \\
\hline Trombose venosa profunda & 3 & 1,9 \\
\hline Sangramento & 3 & 1,9 \\
\hline Alteração hemodinâmicas & 2 & 1,3 \\
\hline Alteração de saturação & 2 & 1,3 \\
\hline Parada cardíaca & 2 & 1,3 \\
\hline Perda de consciência & 2 & 1,3 \\
\hline Complicações respiratórias e cirúrgicas & 2 & 1,3 \\
\hline Diminuição do fluxo sanguíneo & 2 & 1,3 \\
\hline Baixa perfusão & 2 & 1,3 \\
\hline Hipóxia & 2 & 1,3 \\
\hline Retardo & 2 & 1,3 \\
\hline Outros* & & 24,8 \\
\hline Not * & \\
\hline
\end{tabular}

Nota: *Outros sinais com frequência unitária.

principal forma de diminuição da temperatura corporal ${ }^{(12)}$.

Sobre os momentos importantes para se realizar o controle da temperatura corporal do paciente, a maioria dos entrevistados relata corretamente que esta deve ser feita em todos os momentos do perioperatório. Ressalta-se que a manutenção da temperatura em níveis normais é importante para evitar complicações e reduzir o tempo de permanência do paciente na SRPA, bem como pode repercutir na diminuição da necessidade de transfusões e no custo com administrações de medicações e solicitação de exames laboratoriais adicionais ${ }^{(13)}$.

Dentre os sinais que indicam a presença de hipotermia, pode-se inferir que neste as- pecto grande parte desconhece que a queda da temperatura pode se tornar complexa e apresentar outros sinais à medida que varia a sua gravidade. Nos casos mais extremos, por exemplo, é possível ocorrer a perda dos reflexos oculares, oligúria, edema pulmonar, arreflexia, apneia, coma e assistolia ${ }^{(14)}$.

Sobre os fatores relacionados ao paciente, ao ambiente e ao procedimento cirúrgico que podem contribuir para a ocorrência da hipotermia, os mais citados pela equipe foram o tipo de anestesia, fator determinante principalmente se for combinada, o tempo e o tipo de cirurgia que são elementos que afetam a temperatura, tornando-a menor quanto maior for o tempo, e a idade do paciente. Em extremos de idade, como ao nascer, o paciente possui um mecanismo de regulação térmica imaturo, enquanto no idoso isso acontece devido à diminuição de tecido subcutâneo, do metabolismo e falhas no mecanismo de controle vasomotor ${ }^{(12,13)}$.

Outros fatores desconhecidos pela maioria da equipe e que podem ser elencados são: o nível hormonal, que afeta principalmente mulheres; o uso de drogas e medicamentos que podem interferir na capacidade termorreguladora do organismo; e o ritmo circadiano, que pode fazer o corpo apresentar variações de $0,5{ }^{\circ} \mathrm{C}$ a $1,0^{\circ} \mathrm{C}$ durante o período de 24 horas $^{(12,13)}$.

Sobre os dispositivos conhecidos para aquecer o paciente, a equipe mostra conhecer alguns que não estão comumente disponíveis no serviço, como colchões, mantas térmicas e botas. Entre as principais medidas a serem tomadas para manutenção da normotermia dos pacientes destacam-se: os métodos passivos com aquecimento por meio de exposição limitada da pele e uso de cobertores de algodão, e os métodos mais eficazes de aquecimento ativo por meio do uso de colchões térmicos, infusão de líquidos aquecidos, aquecimento e umidificação dos gases administrados s, $10,15,16)^{2}$. Além dessas medidas, recomenda-se o aumento da temperatura da sala cirúrgica quando o aquecimento ativo não é viável ou não é suficiente para aquecer o paciente ${ }^{(17)}$.

Sobre as possíveis complicações decorrentes da hipotermia perioperatória, as mais extremas foram citadas, como a parada cardiorrespiratória e o óbito. No entanto, isoladamente alguns profissionais mencionam diversas outras complicações. A exacerbação da dor pós-operatória de fato acontece com a hipotermia, assim como a taquicardia, aumento da demanda de oxigenação, o rebaixamento do nível de consciência pelo aumento da meia-vida dos anestésicos, a diminuição do débito urinário, e alterações e complicações hemodinâmicas como a trombose venosa profunda, pela estase venosa ${ }^{(13)}$.

De maneira geral, é possível perceber que os participantes da pesquisa possuem um conhecimento básico sobre a hipotermia no paciente perioperatório, principalmente sobre o conceito, os sinais, alguns sintomas 
e como intervir quando o paciente apresenta o problema. Entretanto, este conhecimento precisa ser mais aprofundado quanto à sua etiologia e suas possíveis complicações, o qual pode ser realizado por meio de educação permanente e refletirá positivamente nas intervenções e assistência.

É essencial que a equipe de enfermagem realize a avaliação pré-operatória a fim de facilitar a identificação de pacientes em risco de desenvolver hipotermia, pois o custo para a prevenção é menor que o custo necessário para tratar seus resultados adversos. Para isso, o enfermeiro exerce um papel crucial no planejamento e implementação de intervenções que minimizem os riscos e proporcionem o tratamento adequado da hipotermia perioperatória, garantindo a qualidade da assistência ao paciente cirúrgico ${ }^{(1,18,19)}$.

\section{CONCLUSÃO}

A hipotermia é um problema clínico comum, o que exige do profissional de enfermagem compreensão e conhecimento sobre este evento adverso. Tal competência reduz a possibilidade de complicações durante o ato cirúrgico e no pós-operatório, além de repercutir na melhoria da assistência, diminuição de custos para a instituição, redução do tempo de permanência na SRPA e uma rápida recuperação cirúrgica.
Esta assistência segura é garantida por intermédio de uma formação qualificada dos profissionais de enfermagem e exige políticas de educação permanente e continuada nos serviços de saúde. Assim, a manutenção da atualização profissional possibilita atuação em diversas situações e desenvolvimento das habilidades necessárias para o manejo da hipotermia.

A hipotermia perioperatória é um assunto que apresenta lacunas de conhecimento e intervenções de enfermagem e espera-se que os achados desse estudo auxiliem no planejamento de ações educativas que norteiem as estratégias de assistência de qualidade ao paciente cirúrgico.

\section{Referências}

1. Pereira NHC, Rocha ADM, De Mattia AL. Infusão venosa aquecida relacionada à prevenção das complicações da hipotermia intraoperatória Rev. SOBECC [Internet]. 2014 abr./jun.;19(2): 74-78. DOI: http://doi.org/10.4322/ sobecc.2014.013.

2. Silva ED, Mendes FF, Braz LG, et al. Instruç̃̃es brasileiras sobre intervenções para prevenção e treinamento a respeito de hipotermia perioperatória inadvertida em adultos-produzida pela Sociedade de Anestesiologia do estado de São Paulo. Journal of Infection Control [Internet]. 2018 [acesso em $30 \mathrm{abr}$ 2020]; 7(1):3-18. Disponível em: http://jic-abih.com.br/index.php/jic/article/ view/218/pdf.

3. Uriostegui-Santana ML, Nava-López JA, Mendoza-Escoto VM. Alteraciones de la temperatura y su tratamiento en el perioperatorio. Revista Mexicana de Anestesiología [Internet]. 2017 [acesso em 30 abr 2020]; 40(1):29-37. Disponivel em: https://www.medigraphic.com/pdfs/rma/cma-2017/cma171 e.pdf. 4. Healy K, O'Sullivan A, McCarthy L. A nurse-led audit on the incidence and management of inadvertent hypothermia in an operating theatre department of an Irish hospital. Journal of perioperative practice [Internet]. 2019; 29(3):54-60. DOl: http://doi.org/10.1177/1750458918793295.

5. Giuliano KK, Hendricks J. Inadvertent perioperative hypothermia: Current nursing knowledge. AORN journal [Internet]. 2017; 105(5):453-463. DOI: http://doi.org/10.1016/j.aorn.2017.03.003.

6. Campbell G, Alderson P, Smith AF, Warttig S. Warming of intravenous and irrigation fluids for preventing inadvertent perioperative hypothermia. Cochrane Database Syst Rev. 2015; 4:CD009891. DOl: http://doi. org/10.1002/14651858.CD009891.

7. Sanguiné AS, Ramos GFN, Boschetti JR, Treviso P. Hipotermia no pós-operatório imediato. REV. SOBECC. 2018; 23(4):205-211. DOI: https://doi. org/10.5327/Z1414-4425201800040006.

8. Silva ECL, Cardoso GJP, Oliveira JAA, Costa PPS, et al. Ações de enfermagem no controle da hipotermia em pacientes no transoperatório. In: $11^{\circ}$ Simpósio Internacional de Esterilização e Controle de Infecção relacionada à saúde. Anais Eletronicos [Internet]. 2018 [acesso em 30 abr 2020]. Disponível em: https://proceedings.science/sobecc-2018/papers/as-acoes-de-enfermagem-no-controle-da-hipotermia-em-pacientes-no-transoperatorio.

9. Maurício $\mathrm{Fl}$, et al. Fatores De Risco Para 0 Desencadeamento Da Hipotermia No Intraoperatório: Um Estudo Bibliográfico. CORPVS, 2015; 1 (25): 26-31. Disponível em: http://publica-estaciofic.com.br/revistas/index.php/CORPVS/ article/view/48

10. Danczuk RFT, Nascimento ERP, Silveira NR, Hermida PMV, Rasia MA. Mé- todos de aquecimento na prevenção da hipotermia no intraoperatório de cirurgia abdominal eletiva. Esc Anna Nery [Internet]. 2015; 19(4):578-584. DOI: http://dx.doi.org/10.5935/1414-8145.20150077.

11. Mendoza IYQ, Peniche ACG. Intervenção educativa sobre hipotermia: uma estratégia de ensino para aprendizagem em Centro Cirúrgico. Rev Esc Enferm USP [Internet]. 2012; 46(4):851-857. DOI: http://dx.doi.org/10.1590/S008062342012000400010 .

12. Amante LN, Slomochenski LA, Teixeira MGPN, Bertoncello KCG. Ocorrência de hipotermia não planejada em sala de recuperação anestésica. UNOPAR Cient, Ciênc Biol Saúde [Internet]. 2012. DOI: http://dx.doi.org/10.17921/ 2447-8938.2012v14n4p\%25p.

13. Moysés AM, Trettene AS, Navarro LHC, Ayres JA. Prevenção da hipotermia no transoperatório: comparação entre manta e colchão térmicos. Rev Esc Enferm USP [Internet]. 2014; 48(2):228-235. DOI: http://dx.doi.org/10.1590/ S0080-623420140000200005.

14. Martins LP, Junior NO, Riegel F, Arregino DS. The nursery against the complications of hypothermia in the immediate post-operative. Revista de Enfermagem da UFPI [Internet]. 2019 [acesso em 30 abr 2020]; 8(1):68-73. Disponível em: https://revistas.ufpi.br/index.php/reufpi/article/view/7700.

15. Echeverry-Marin PG, Rincón-Valenzuela DA, Monroy-Charry AG, Ruiz-Villa JO, Higuera-Redondo G, Rubio-Elorza JH. Survey about attitudes on perioperative temperature monitoring and thermal protection in Colombia. Colombian journal of anesthesiology [Internet]. 2016 [acesso em 30 abr 2020]; 44(4):282-291. Disponível em: https://link.springer.com/chapter/10.1007/978-3-030-03143-53.

16. Ribeiro E., Navarro NT, Armede VCB, Rodrigues HS, Valle JP, Duran ECM. Frequência de hipotermia não intencional no perioperatório de cirurgias eletivas. Revista SOBECC [Internet]. 2016 [acesso em 30 abr 2020]; 21(2):68-74. Disponível em: https://revista.sobecc.org.br/sobecc/article/view/15.

17. Association of Operating Room Nurses. Recommend practices for the prevention of unplanned perioperative hypothermia. AORN J [Internet]. 2007; 85(5):972-88. DOI: https://doi.org/10.1016/j.aorn.2007.04.015.

18. Guedes LI, Sousa MAM, Abreu SAL, Batista AIM. Prevenir a hipotermia no perioperatório: revisão integrativa da literatura. Rev. Enf. Ref. [Internet]. 2015. DOI: http://dx.doi.org/10.12707/RIV14027.

19. Penaforte HSC, Seara L, Costa MJ, Mendes A. Normotermia no perioperatório: perspectiva do enfermeiro. Revista de Investigação \& Inovação em Saúde [Internet]. 2019 [acesso em 30 abr 2020]; 2(1):7-17. Disponível em: https://www.essnortecvp.pt/upload/files/maquetizacao7.pdf. 